\title{
Intracellular virus sensor MDA5 exacerbates vitiligo by inducing the secretion of chemokines in keratinocytes under virus invasion
}

Tongtian Zhuang ${ }^{1}$, Xiuli Yi ${ }^{1}$, Jianru Chen ${ }^{1}$, Pan Kang ${ }^{1}$, Xuguang Chen ${ }^{1}$, Jiaxi Chen', Tingting Cui , Yuqian Chang ${ }^{1}$,

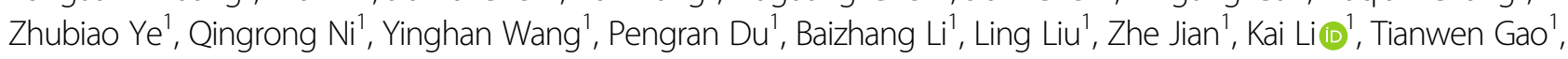
Shuli $\mathrm{Li}^{1}$ and Chunying $\mathrm{Li}^{1}$

\begin{abstract}
Vitiligo is a disfiguring disease featuring chemokines-mediated cutaneous infiltration of autoreactive $C D 8^{+} T$ cells that kill melanocytes. Copious studies have indicated that virus invasion participates in the pathogenesis of vitiligo. $|F| H 1$, encoding MDA5 which is an intracellular virus sensor, has been identified as a vitiligo susceptibility gene. However, the specific role of MDA5 in melanocyte death under virus invasion is not clear. In this study, we first showed that the expression of anti-CMV IgM and MDA5 was higher in vitiligo patients than healthy controls. Then, by using Poly $(I: C)$ to imitate virus invasion, we clarified that virus invasion significantly activated MDA5 and further potentiated the keratinocyte-derived CXCL10 and CXCL16 which are the two vital chemokines for the cutaneous infiltration of $\mathrm{CD}^{+} T$ cells in vitiligo. More importantly, IFN- $\beta$ mediated by the MDA5-MAVS-NF-KB/IRF3 signaling pathway orchestrated the secretion of CXCL10 via the JAK1-STAT1 pathway and MDA5-meidiated IRF3 transcriptionally induced the production of CXCL16 in keratinocytes under virus invasion. In summary, our results demonstrate that MDA5 signaling orchestrates the aberrant skin immunity engaging in melanocyte death via mediating CXCL10 and CXCL16 secretion, which supports MDA5 as a potential therapeutic target for vitiligo under virus invasion.
\end{abstract}

\section{Introduction}

Vitiligo is a disfiguring disease characterized by melanocyte death which is caused by autoreactive melanocytespecific $\mathrm{CD}^{+} \mathrm{T}$ cells with the attraction of excessive chemokines $^{1,2}$, which underlines the autoimmunity dominantly participates in the pathogenesis of vitiligo. Multiple studies showed that vitiligo is caused by specific environmental factors in the population with genetic predisposition $^{3}$. Virus, as an important environmental factor, has been determined to play profound parts in the

\footnotetext{
Correspondence: Shuli Li (lishli@fmmu.edu.cn) or Chunying Li (lichying@fmmu. edu.cn)

'Department of Dermatology, Xijing Hospital, Fourth Military Medical University, No 127 of West Changle Road, Xi'an, Shaanxi 710032, China These authors contributed equally: Tongtian Zhuang, Xiuli Yi, Jianru Chen Edited by E. Candi
}

pathogenesis of many autoimmune diseases including type 1 diabetes ${ }^{4-6}$, rheumatic arthritis ${ }^{7-9}$, and autoimmune thyroid disease $\mathrm{e}^{10,11}$. These autoimmune diseases all occur with increased frequency in vitiligo patients ${ }^{12,13}$. In vitiligo paraffin-embedding tissues, the detective rate of CMV DNA is markedly higher than that in healthy controls $(38 \% \text { vs } 0)^{14}$. Besides, the case-control study based on the large sample size determines that hepatitis $C$ virus $(\mathrm{HCV})^{15}$ is associated with vitiligo. Additionally, A strong causative link between turkey herpesvirus and vitiligo has also been established in the Smyth line chicken ${ }^{16}$. Moreover, many cases embody the correlation between virus infection and vitiligo ${ }^{17-22}$, particularly the inflammatory raised borders in vitiligo with $\mathrm{HCV}$ infection ${ }^{19}$, the reactivation of herpes simplex virus in lesions of vitiligo after excimer laser treatment ${ }^{20}$ and vitiligo occurrence after the 
resolution of drug-induced hypersensitivity syndrome which is characterized by sequential reactivations of herpes viruses ${ }^{21,22}$. It is noted that interferon-induced helicase C domain 1 (IFIH1), encoding melanoma differentiation-associated 5 protein (MDA5) which could sense the invasion of virus, has been identified as a susceptible gene for vitiligo by several genome-wide association studies (GWAS) ${ }^{23-25}$. However, the specific role of MDA5 in the pathogenesis of vitiligo under virus invasion is not clear.

As a pathogen pattern recognition receptor (PRR), MDA5 could sense the virus replication intermediate dsRNA derived from no matter DNA or RNA virus ${ }^{26,27}$, and Poly(I:C) is an analog of this kind of dsRNA ${ }^{28}$. After sensing invasion of viruses, MDA5 triggers potent signaling cascade to eradicate virus by initiating the type I interferon (IFNs) ${ }^{29}$. Meanwhile, the inappropriate activation of MDA5 increases the risk of autoimmune diseases $^{5,30-33}$. Upon activated, MDA5 could exploit the longer dsRNA ( $>1 \mathrm{~kb})$ as a scaffold to induce the polymerization of the adaptor mitochondrial antiviral signaling protein (MAVS), a protein anchored in mitochondrial outer membrane ${ }^{34-36}$. Polymerization of MAVS triggers a signaling cascade culminating in the activation of the interferon-regulating factor 3 (IRF3) and the nuclear factor-kappa $\mathrm{B}(\mathrm{NF}-\mathrm{kB})^{37,38}$. These transcription factors induce the type I and III IFNs, as well as other cytokines or chemokines ${ }^{39-42}$.

In vitiligo, the excessive CXCL10 (chemokine (C-X-C motif) ligand 10$)^{43,44}$ and CXCL16 $6^{45}$ in epidermis have been established to play key roles in the skin trafficking of autoreactive melanocyte-specific $\mathrm{CD}^{+} \mathrm{T}$ cells ${ }^{1}$, which has been recognized as the main cause of melanocyte death in vitiligo. Thus, we hypothesized that intracellular virus sensor MDA5 could exacerbate the melanocyte death in vitiligo by inducing the section of chemokines from keratinocytes under virus invasion.

In the present study, we initially manifested that the expression of MDA5 and anti-CMV IgM was upregulated in some progressive vitiligo, which was accompanied by the increment of the mRNA levels of CXCL10, CXCL16 in the epidermis and the infiltration quantity of $\mathrm{CD}^{+} \mathrm{T}$ in the skin tissues. Then, we clarified that virus invasion significantly activated MDA5 and further potentiated the secretion of CXCL10 and CXCL16 in keratinocytes. Mechanistically, we revealed that IFN- $\beta$ mediated by the MDA5-MAVS-NF- $k B /$ IRF3 signaling pathway orchestrated the secretion of CXCL10 via the JAK1-STAT1 pathway and MDA5meidiated IRF3 transcriptionally induced the production of CXCL16 in keratinocytes under virus invasion. Therefore, we supported the MDA5 signaling pathway as a promising therapeutic target in vitiligo under virus invasion.

\section{Materials and methods}

\section{Patients and samples}

The peripheral blood samples and skin samples of vitiligo were collected from patients given a diagnosis of vitiligo at a progressive stage according to clinical manifestation in the Department of Dermatology, Xijing Hospital of the Fourth Military Medical University. All subjects had not received any systematic or topical therapy within 3 months before serum and skin samples collection. The control blood samples were collected from age- and sex- matched voluntary staffs working in Xijing hospital and the control skin samples collected from the age- and sex- matched subjects during plastic surgery in Xijing hospital. The skin samples of atopic dermatitis and psoriasis were collected from the in-patient given a diagnosis of atopic dermatitis and psoriasis respectively in the Department of Dermatology, Xijing Hospital of the Fourth Military Medical University. All participants gave their written informed consents according to the Declaration of Helsinki and approval from Clinical Research Ethics Committee of Xijing Hospital, Fourth Military Medical University. All serum samples were stored at $-80^{\circ} \mathrm{C}$ without repeated freezing and thawing. All skin samples used for immunofluorescence assay were embedded in paraffin at $4{ }^{\circ} \mathrm{C}$. The total protein for Western-blot assay was extracted from fresh skin samples immediately and was stored at $-20^{\circ} \mathrm{C}$. The total RNA for qRT-PCR assay was extracted from fresh skin samples and reverse-transcribed to cDNA and stored at $-20^{\circ} \mathrm{C}$. (The numbers of subjects used for each assay were listed in Supplementary Table 1).

\section{Cytomegalovirus IgM detection}

The cytomegalovirus IgM detection enzyme-linked immunosorbent assay (ELISA) kit (Abcam, ab108725, USA) was used to detect the anti-CMV IgM in serum according to the manufacturer's instructions. The details are in the Supplementary methods.

\section{Virus invasion simulation}

Poly $(\mathrm{I}: \mathrm{C})$ is a synthetic analog of double-stranded RNA (dsRNA), a molecule pattern associated with viral infection. The Poly $(\mathrm{I}: \mathrm{C})$ used in this article is Poly(I:C)/Lyo$\mathrm{Vec}^{\mathrm{TM}}$ (Invivogen, USA) which are performed complexes between Poly(I:C) and the transfection reagent $\mathrm{LyoVec}^{\mathrm{TM}}$ which can ensure that it functions in cytosol. Combined with the characteristic that they have an average size of $1.5-8 \mathrm{~kb}$, these complexes could imitate the virus infection and further be sensed by MDA5.

\section{Cell culture}

Normal Human keratinocytes (NHKs) were isolated from healthy subjects and cultured in keratinocyte-SFM (Gibco, USA). Cells were grown to $80 \%$ confluency and 
used in 3-5 passages. A human keratinocyte cell line was purchased from American Tissue Culture Collection (ATCC) and cultured in Modified Medium RPIM 1640 with 10\% FBS and L-glutamine (Invitrogen, USA). Poly(I: C) (Invivogen, USA) was added to culture at different concentrations $(0.3 \mu \mathrm{g} / \mathrm{ml}, 0.6 \mu \mathrm{g} / \mathrm{ml})$ in dose-dependent assays and at $0.6 \mu \mathrm{g} / \mathrm{ml}$ in function and signaling pathway exploration assays. For experiments, supernatant was harvested for ELISA, and the cells were processed for quantitative real-time PCR (qRT-PCR), Western-blot and immunofluorescence assay at the indicated time points.

\section{Annexin V-PE/7AAD apoptosis assay}

NHKs were plated into 6-well plates at the density of $3 \times 10^{5}$ cells/well and treated with Poly(I:C) within $1.5 \mu \mathrm{g} /$ $\mathrm{ml}$ for $24 \mathrm{~h}$. Cell apoptosis was detected by using the Annexin V-PE/7-AAD kit (MultiSciences, China). Annexin $\mathrm{V}-\mathrm{PE}$ and 7AAD fluorescence were measured using flow cytometry (Beckman Coulter, USA) and analyzed with Expo32 software (Beckman Coulter, USA).

\section{Immunofluorescence assay}

For skin samples embedded with paraffin of progressive vitiligo patients with positive or negative anti-CMV IgM and healthy controls, the samples were deparaffinized and boiled in antigen retrieval solution $(\mathrm{PH}=9.0)$. Next, the sections were incubated with the normal goat serum (BosterBio, USA) for $1 \mathrm{~h}$ after 3 times washing with PBS. Then, incubated with the primary antibody (rabbit monoclonal anti-MDA5, Abcam, ab126630, USA; rabbit polyclonal anti-IFN- $\beta$, Proteintech, 27506-1-AP, China; rabbit monoclonal anti-CD8 $\alpha$, Abcam, ab108343, USA) overnight at $4{ }^{\circ} \mathrm{C}$. After washing, the secondary antibody (goat anti-rabbit IgG-Alexa Fluor 488 Conjugated, zhuangzhiBIO, China) was used for $1 \mathrm{~h}$ at room temperature in dark. The cell nuclei were stained with Hoechst 33258 (Sigma-Aldrich, German) for $10 \mathrm{~min}$ in dark. Immunofluorescence analysis was applied with laser scanning confocal microscopy (Olympus, Japan).

For cell immunofluorescence, the HaCaT cells or NHKs were cultured on culture plates and treated with $0.6 \mu \mathrm{g} / \mathrm{ml}$ $\operatorname{Poly}(\mathrm{I}: \mathrm{C})$ for the indicated time. Then, they were fixed with $4 \%$ paraformaldehyde for $10 \mathrm{~min}$. After washing, the cells were incubated with $0.1 \%$ Triton X-100 for $10 \mathrm{~min}$ at room temperature, and then were blocked with normal goat serum for $30 \mathrm{~min}$. The following procedures were similar as the histological immunofluorescence assay with the use of primary antibodies (rabbit monoclonal antiMDA5, Abcam, ab126630, USA; mouse monoclonal antiMAVS, Abcam, ab220170, USA) and the secondary antibodies (goat anti-rabbit IgG-Alexa Fluor 488 Conjugated, zhuangzhiBIO, China; goat anti-mouse IgG-Cy3 Conjugated, zhuangzhiBIO, China).

\section{Determination of IC50 by CCK8 assay}

Roughly, the 50\% inhibitory concentration (IC50) of Poly(I:C) on NHKs was determined by CCK8 kit (7Sea biotech, China) according to the manufacturer's instructions. In general, seeded in 96-well culture plates, cells were treated with $0-4 \mu \mathrm{g} / \mathrm{ml}$ Poly(I:C) for $24 \mathrm{~h}$. Next, incubated with $100 \mu \mathrm{l}$ fresh medium with $10 \mu \mathrm{l}$ CCK8 solution for $2 \mathrm{~h}$ at $37^{\circ} \mathrm{C}$ and optical density (OD) was measured at $450 \mathrm{~nm}$ by Model 680 Microplate Reader (Bio-Rad, USA).

\section{Western-blot assay}

After washed and lysed, the total protein was collected and the concentration was measured with BCA Protein Assay kit (Pierce, USA). Equal amounts of protein were separated by $10 \%$ SDS-PAGE (Bio-Rad, USA) and transferred to Polyvinylidene difluoride membranes (Millipore, USA). Then the molecules were blocked with $5 \%$ non-fat dry milk for $2 \mathrm{~h}$. After washed with TBST transitorily, the membranes were incubated with the corresponding primary antibodies (Supplementary Table 2) overnight at $4{ }^{\circ} \mathrm{C}$. After washing, the membranes were incubated with secondary antibodies (horseradish peroxidase-labeled goat anti-rabbit IgG, Santa Cruz, USA; horseradish peroxidase-labeled goat anti-mouse IgG, Santa Cruz, USA) for $1 \mathrm{~h}$ at room temperature. The bands were detected by ChemiDocTM XRS + system (Bio-Rad, USA). As for the determination of MDA5 expression in skin tissues by Western-blot, the skin samples were soaked in Dispase at $4{ }^{\circ} \mathrm{C}$ overnight, and then we removed the dermis and subcutaneous tissues to get the epidermis tissues. The remaining procedures were similar to the above description.

\section{Small interfering RNA (siRNA) transfection}

Cells were seeded at $3 \times 10^{5}$ cells/well for $24 \mathrm{~h}$ before the transfection. The $6 \mu \mathrm{l}$ corresponding siRNA (IFIH1 siRNA,5'-GCACGAGGAAUAAUCUUUATT-3' GenePharma China; MAVS siRNA, 5'-CACAGGGUCAG UUGUAUCUTT-3' GenePharma China; NC siRNA, 5'UUCUCCGAACGUGUCACGUTT-3' GenePharma China; NF-kB siRNA Santa Cruz, sc29410, USA; IRF3 siRNA Santa Cruz, sc35710, USA) and $6 \mu$ l Lipofectamine 3000 (Invitrogen, USA) were added to $250 \mu 1640$ medium without fetal bovine serum (FBS). After mixed evenly, it was placed at room temperature for $10 \mathrm{~min}$. Then, the mixed liquid was added to each well with $2 \mathrm{ml} \mathrm{FBS-containing} \mathrm{fresh}$ medium.

\section{RNA isolation and qRT-PCR}

Total RNA was extracted with TRIzol (Ambion, USA) and then reverse-transcribed to cDNA using a PrimeScript RT reagent kit (Takara, Japan). qRT-PCR was performed by using SYBR Premix Ex Taq II (Takara, 
Japan) with the iQ5 PCR Detection System (Bio-Rad, USA). Threshold cycle (CT) values were used to calculate the fold change by the $2^{-\Delta \Delta C T}$ method. The relative mRNA expression was normalized to $\beta$-actin. Primer sequences are listed in Supplementary Table 3.

\section{ELISA assay}

Human CXCL9, CXCL12, CCL26, CCL2, IL-15, IFN- $\beta$ Quantikine ELISA kits (Elabscience, China) and CXCL10, CXCL16, CCL20 DuoSet ELISA kits (R\&D Systems, USA) were used to analyze cell supernatant samples, according to the corresponding manufacturer's instructions.

\section{Semi-denaturing detergent agarose gel electrophoresis (SDD-AGE)}

Semi-denaturing detergent agarose gel electrophoresis (SDD-AGE) was conducted according to the published protocol $^{36}$. Crude cell lysis was resuspended in $1 \times$ sample buffer $(0.5 \times \mathrm{TBE}, 10 \%$ glycerol, $2 \%$ SDS, and $0.0025 \%$ bromophenol blue) and loaded onto a vertical 1.5\% agarose gel (Bio-Rad, USA). After electrophoresis in the running buffer ( $1 \times \mathrm{TBE}$ and $0.1 \% \mathrm{SDS})$ for $35 \mathrm{~min}$ with a constant voltage of $100 \mathrm{~V}$ at $4{ }^{\circ} \mathrm{C}$, the proteins were transferred to Immobilon membrane (Millipore, USA) for immunoblotting and the following procedures are same as the Western-blot assay.

\section{Chromatin immunoprecipitation assay}

Chromatin immunoprecipitation analysis was performed by using the ChIP (Chromatin immunoprecipitation) Assay Kit (Millipore, USA), according to the manufacturer's instructions. Briefly, $\mathrm{HaCaT}$ cells were cross-linked with DNA by using $1 \%$ formaldehyde at room temperature for $10 \mathrm{~min}$. After washing, cells were lysed in SDS lysis buffer. DNA was sheared to small fragments of less than $500 \mathrm{bp}$ in length by means of sonication. The recovered supernatant fraction was incubated with antibodies overnight on a rotor at $48^{\circ} \mathrm{C}$. After washing, the precipitated IRF3 protein-DNA complexes were recovered by using IRF3 antibodies and protein $\mathrm{G}-\mathrm{Sepharose}$ beads at $48^{\circ} \mathrm{C}$ for $16 \mathrm{~h}$. DNA was then purified with phenol/chloroform. A fraction was used as the PCR template (forward, 5'-CAGCCC TGGGTTCTTACCACTC-3'; reverse, 5'-AGGATGG CTGCCGAGAGGAC- $3^{\prime}$ ) to detect the presence of promoter sequences between -1253 and -1338 of CXCL16. After the PCR amplification, each PCR reaction was analyzed by $2 \%$ agarose gel electrophoresis with D2000 DNA ladder (Solarbio, China).

\section{Statistical analysis}

Each statistical analysis was performed by GraphPad Prism 7 for Windows (USA) with two-tailed Student's unpaired t-tests or one-way analysis of variance
(ANOVA). These data are conformed to the normal distribution and variance similarity between the groups that are statistically compared. These data represent as mean \pm SD for at least three independent experiments. " $P<0.05$, ${ }^{* *} P<0.01,{ }^{* * * *} P<0.001,{ }^{\#} P<0.05,{ }^{\# \#} P<0.01,{ }^{\# \#} P<0.001$, ns: not significant.

\section{Results}

The expression of MDA5 and anti-CMV IgM is higher in some progressive vitiligo patients

Previous study has established that the detective rate of CMV DNA in vitiligo paraffin-embedded vitiligo tissues is up to $38 \%{ }^{14}$. To further confirm the link between virus infection and vitiligo, in the serum of progressive vitiligo we first used ELISA to detect anti-CMV IgM, symbolizing acute CMV infection ${ }^{46}$. We found that the positive rate of anti-CMV IgM was $8.92 \%$ in 56 progressive vitiligo patients, while that in 26 healthy controls was $3.84 \%$, and the anti-CMV IgM levels were significantly higher in vitiligo patients than that in healthy controls (Fig. 1a). Of note, the VASI scores ${ }^{47}$ were higher in vitiligo patients with positive anti-CMV IgM than those with negative anti-CMV IgM (Fig. 1b). Consistently, in perilesional epidermis samples, we showed the mRNA levels of CXCL10, CXCL16 (Fig. S1A) in vitiligo patients with positive anti-CMV IgM were higher than that in patients with negative anti-CMV IgM and in healthy controls, also higher than that in the apparently healthy epidermis of vitiligo patients with positive anti-CMV IgM. In parallel, the infiltration quantity of $\mathrm{CD}^{+} \mathrm{T}$ cells in the skin tissues showed the resembled tendency (Fig. S1B). Besides, compared to healthy controls, we showed the higher expression of MDA5 (Fig. 1c, d) and IFN- $\beta$ (Fig. 1e) in perilesional epidermis of vitiligo patients with positive anti-CMV IgM, while no significant upregulation in perilesional epidermis of vitiligo patients with negative anti-CMV IgM and in apparently healthy epidermis of vitiligo patients with positive anti-CMV IgM. Unlike in vitiligo, the expression of MDA5 in the lesional epidermis of psoriasis and atopic dermatitis was not apparently upregulated when compared with that of healthy controls (Fig. S2). Taken together, these data indicated that virus infection correlated with some progressive vitiligo in which MDA5 pathway might play a key role via promoting the secretion of chemokines.

\section{Poly $(\mathrm{l}: \mathrm{C})$ potentiates the expression of MDA5 in vitro}

We further explored how virus invasion influenced the expression of MDA5 by applying Poly(I:C $)^{28,48}$, a kind of virus dsRNA analog simulating the virus replication intermediate regardless of the form of nucleic acid carried by the virion ${ }^{26,27}$. Firstly, IC50 assay and the flow cytometry assay were performed to determine that the safety concentration of Poly(I:C) in NHKs was $0.6 \mu \mathrm{g} / \mathrm{ml}$ 
A

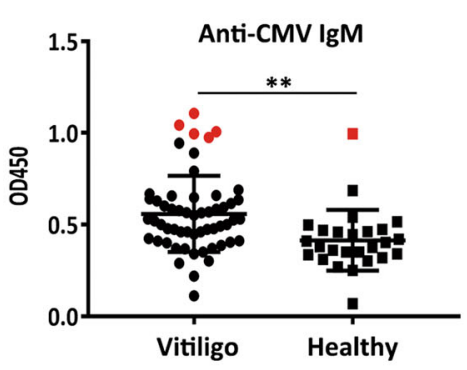

C

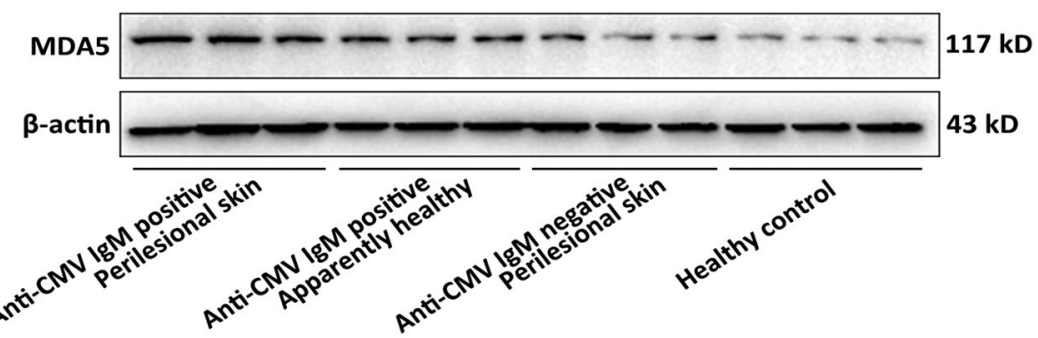

B

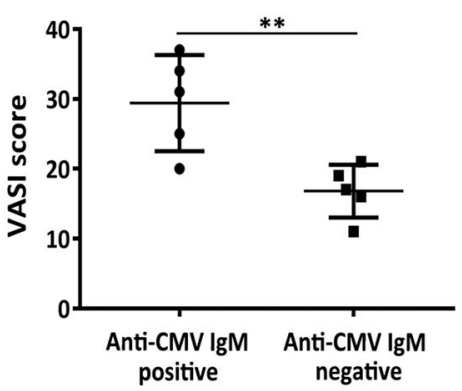

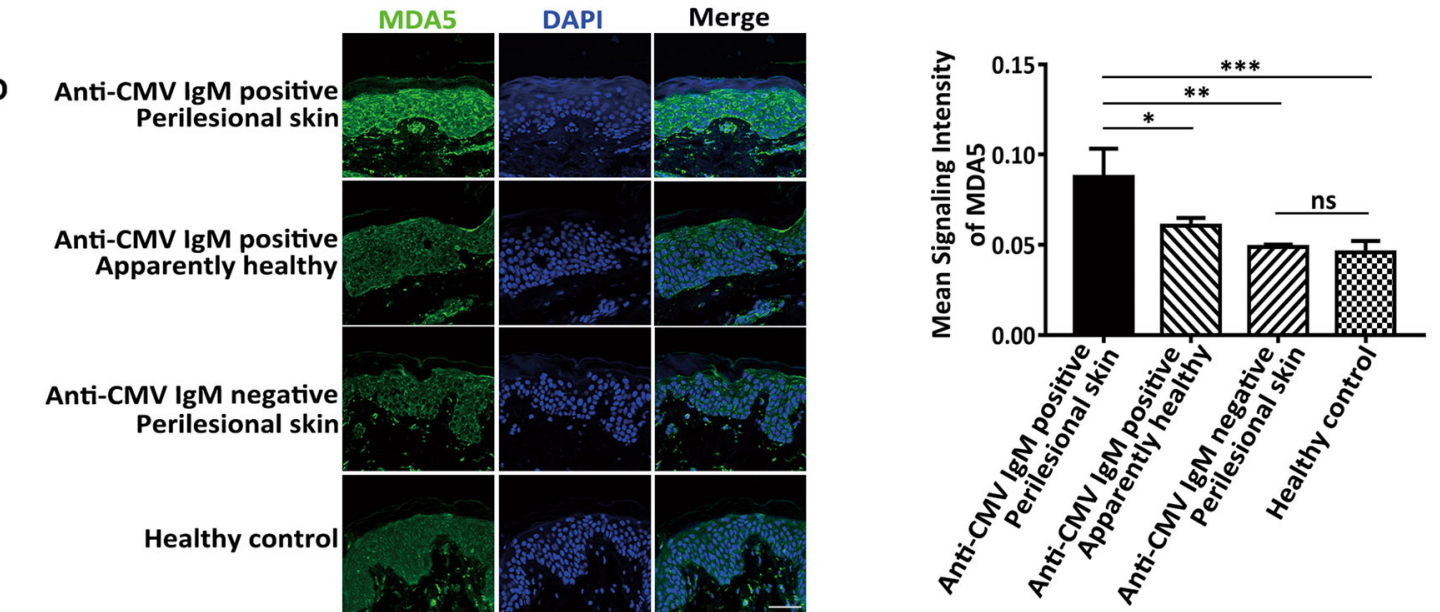

E

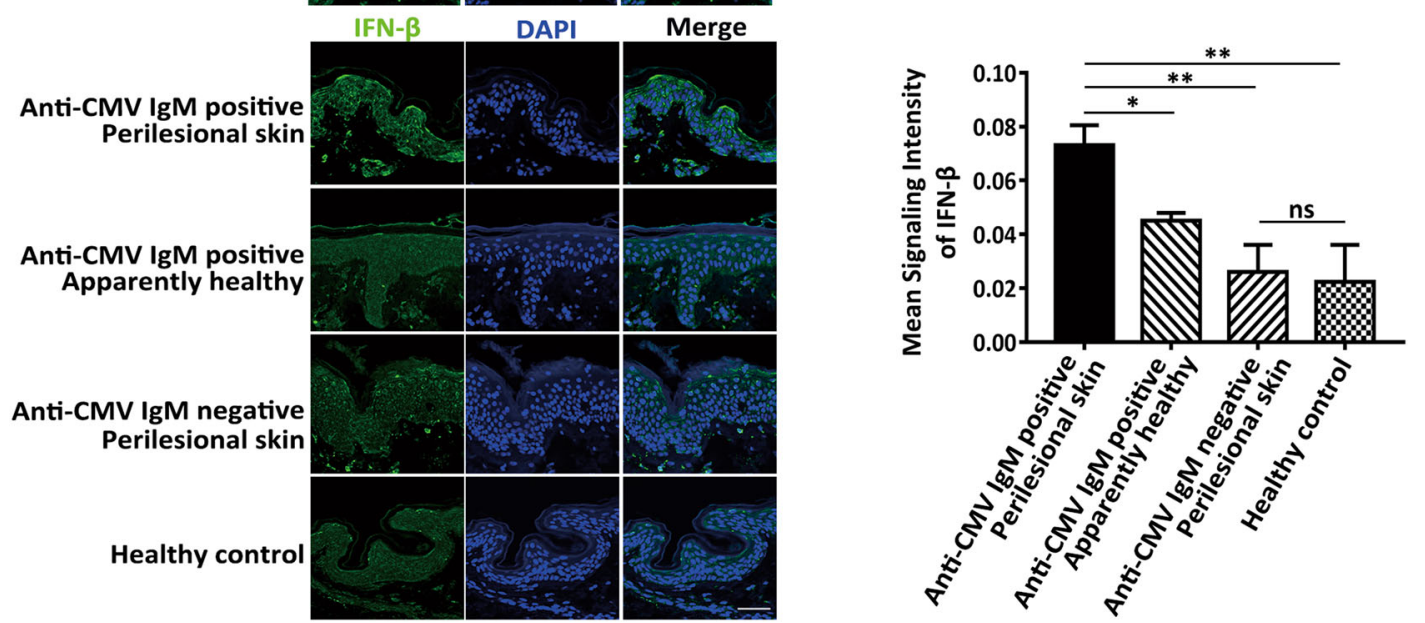

Fig. 1 (See legend on next page.) 
(see figure on previous page)

Fig. 1 The expression of MDA5 and anti-CMV IgM is higher in some progressive vitiligo patients. a The anti-CMV IgM levels in 56 vitiligo patients and 26 healthy controls. The patients with the positive anti-CMV IgM were indicated in red. $\mathbf{b}$ The VASI scores of vitiligo patients with positive anti-CMV IgM or negative anti-CMV IgM. $n=5$. c The Western-blot of MDA5 in the epidermis of perilesional skin of vitiligo patients with positive or negative anti-CMV IgM, in the apparently healthy epidermis of vitiligo patients with positive anti-CMV IgM and in the epidermis of healthy controls. $n=3$. d, e The expression of MDA5 (d) and IFN- $\beta$ (e) in epidermis of perilesional skin of vitiligo patients with positive or negative anti-CMV IgM, in the apparently healthy epidermis of vitiligo patients with positive anti-CMV IgM and in the epidermis of healthy controls, by immunofluorescence assay. $n=5, \operatorname{Bar}=50 \mu \mathrm{m}$. The data are presented as the mean $\pm \mathrm{SD}$ across three independent experiments. ${ }^{*} P<0.05,{ }^{* *} P<0.01$, ${ }^{* * *} P<0.001 . n s$, not significant.

A
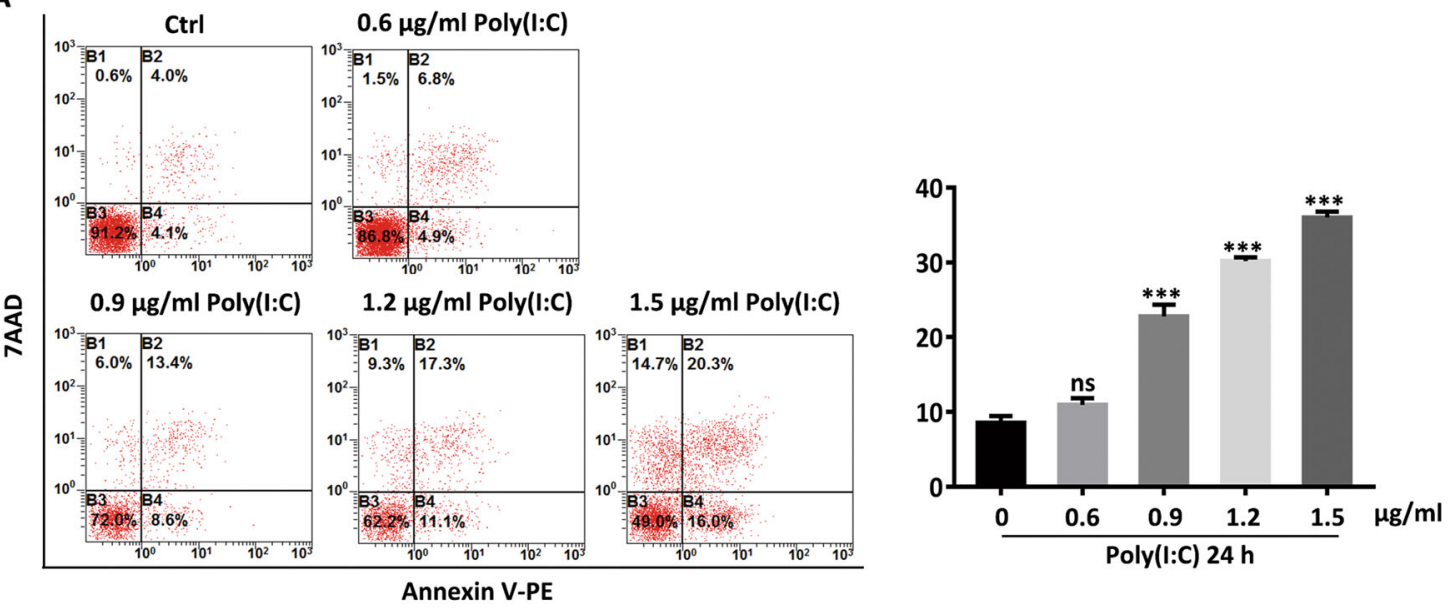

B

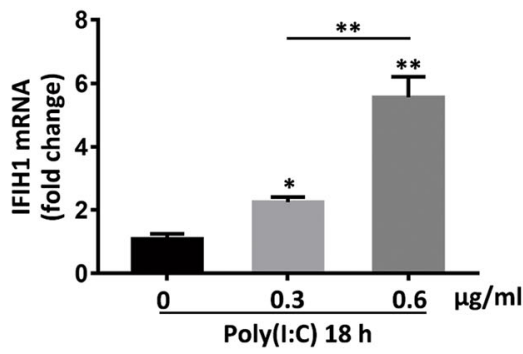

C

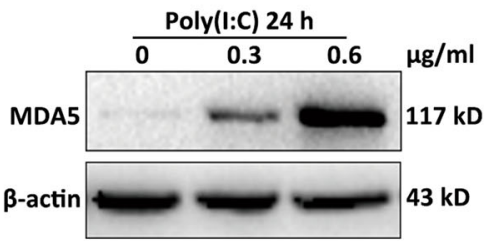

D

E

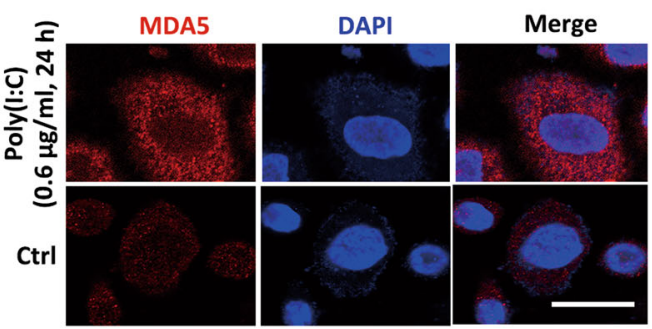

Fig. 2 Poly $(\mathrm{I}: \mathrm{C})$ potentiates the expression of MDA5 in vitro. a The apoptotic rate of NHKs in response to Poly(l:C), detected by flow cytometry assay. b, c The RNA levels (b) and protein levels (c) of IFIH1 in NHKs with Poly(I:C) concentration rose, detected by qRT-PCR assay and Western-blot respectively. $\mathbf{d}$ The expression of MDA5 in NHKs in response to $0.6 \mu \mathrm{g} / \mathrm{ml}$ Poly $(\mathrm{l}: \mathrm{C})$ within $48 \mathrm{~h}$, determined by Western-blot assay. $\mathbf{e}$ The expression of MDA5 in NHKs stimulated by $0.6 \mu \mathrm{g}$ Poly(l:C) for $24 \mathrm{~h}$, detected by immunofluorescence assay. Bar $=10 \mu \mathrm{m}$. The data are representative of three independently performed experiments. ${ }^{*} P<0.05,{ }^{* *} P<0.01,{ }^{* * *} P<0.001$. ns, not significant.

(Fig. S3 and Fig. 2a). Next, the elevated MDA5 mRNA and protein levels were observed in a Poly(I:C) concentrationdependent pattern within $0.6 \mu \mathrm{g} / \mathrm{ml}$ (Fig. 2b, c). In addition, the expression of MDA5 could be enhanced by Poly
(I:C) in a time-dependent way within $24 \mathrm{~h}$, after that the upregulated MDA5 was restored to a basic level gradually (Fig. 2d). Results from immunofluorescence assay reiterated that Poly(I:C) could upregulate the expression of 

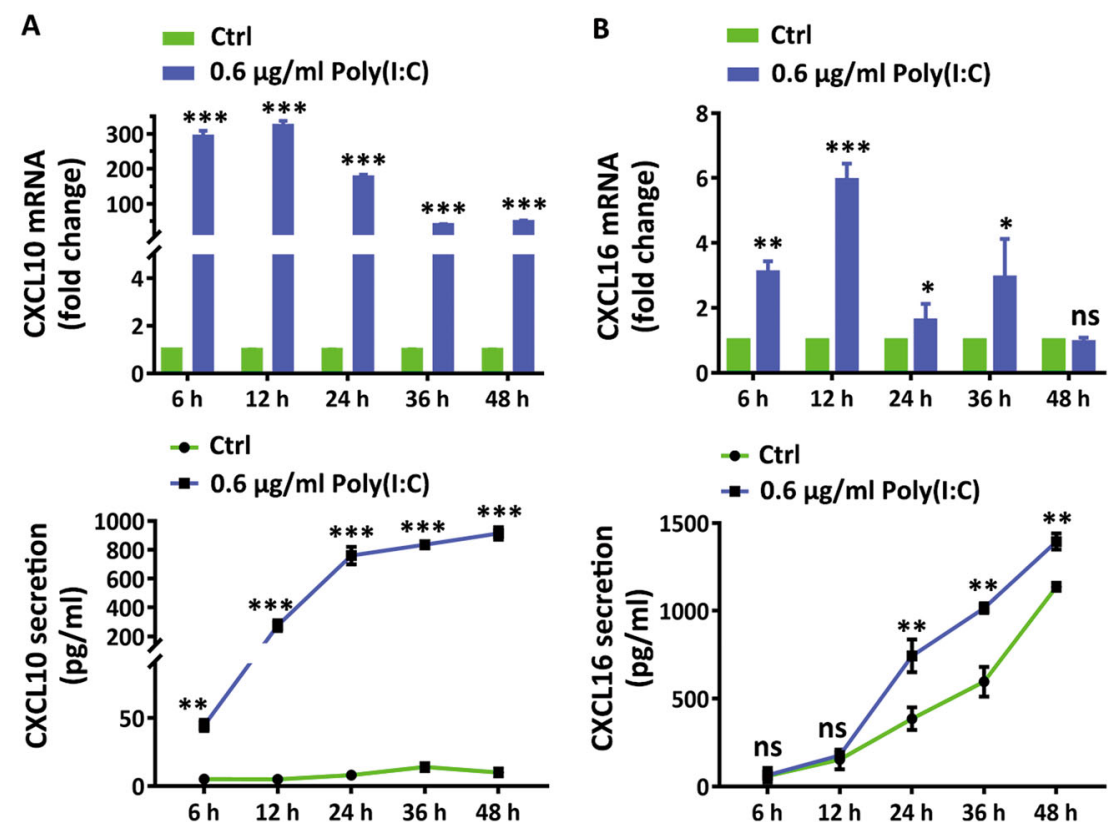

Fig. 3 Poly(l:C) augments the expression of CXCL10 and CXCL16. a The levels of mRNA and secretion of CXCL10 in NHKs in response to $0.6 \mu \mathrm{g}$ Poly $(\mathrm{I}: \mathrm{C})$ at the indicated time points. $\mathbf{b}$ The levels of mRNA and secretion of CXCL16 in NHKs in response to $0.6 \mu \mathrm{g}$ Poly(l:C) at the indicated time points. The data are presented as the mean \pm SD across three independent experiments. ${ }^{*} P<0.05,{ }^{* *} P<0.01,{ }^{* * *} P<0.001$. ns, not significant.

MDA5 (Fig. 2e). Thus, these data manifested Poly(I:C) amplified MDA5 expression in a time- and concentrationdependent way.

\section{Poly $(\mathrm{l}: \mathrm{C})$ augments the secretion of $\mathrm{CXCL} 10$ and $\mathrm{CXCL} 16$}

To understand the impacts of virus invasion on keratinocytes in the pathophysiology of vitiligo, we detected the mRNA alterations of vitiligo-related cytokines and chemokines ${ }^{44,45,49-53}$ in response to Poly(I:C) by qRT-PCR assay. The preliminary results underlined Poly(I:C) induced drastic mRNA increments of CXCL10, CXCL16, CXCL9 and CCL20 (Fig. S4) in a concentrationdependent way. However, no significant influences of Poly(I:C) on the transcription of IL-15, CXCL12, CCL2 and CCL26 (Fig. S4) were observed. Specifically, we detected mRNA and secretion levels of CXCL10 and CXCL16 by qRT-PCR and ELISA respectively, and found that $\operatorname{Poly}(\mathrm{I}: \mathrm{C})$ potentiated the mRNA and secretion levels of CXCL10 and CXCL16 within 48 h (Fig. 3a, b). These data indicated that Poly $(\mathrm{I}: \mathrm{C})$ dictated keratinocytes to generate redundant CXCL10 and CXCL16.

\section{The secretion of CXCL10 and CXCL16 is orchestrated by the combination of MDA5 and MAVS}

Unlike MDA5 functioning potently in an expressionupregulation way, MAVS mainly depends on the formation of aggregates to exert its functions ${ }^{36}$. Consistent with this, we found that the contents of MAVS manifested no obvious change (Fig. 4a) while that of MDA5 significantly mounted in NHKs (Fig. 2c). Alongside, SDD-AGE exhibited formation of MAVS aggregates ${ }^{38}$ following Poly(I:C) stimulation (Fig. 4b) for $18 \mathrm{~h}$ in NHKs. Of note, conspicuous diminution of MAVS aggregates emerged when interfering MDA5 with siRNA (si-MDA5) (Fig. 4b) in $\mathrm{HaCaT}$ cells. Furthermore, the immunofluorescence assay suggested that MDA5, randomly scattering in cytoplasm in stationary phase, colocalized with MAVS swiftly and convened around cell nucleus in response to Poly(I:C) (Fig. 4c). Sequentially, we knocked down MDA5 by using siRNA (Fig. S5A) and found that interfering MDA5 significantly lessened CXCL10 and CXCL16 (Fig. 4d) at both mRNA and secretion levels. Also, interfering MAVS by using siRNA (si-MAVS) had the comparable results (Fig. S5B and Fig. 4e). These data demonstrated that the expression of CXCL10 and CXCL16 is orchestrated by the combination of MDA5 and MAVS with the Poly(I:C) stimulation.

\section{NF-KB and IRF3 mediates the secretion of CXCL10 and CXCL16}

As for IFN- $\beta$ production, previous studies have defined critical roles of NF- $\mathrm{KB}$ and IRF3 as signal transducer in the downstream of MAVS ${ }^{54}$, which provides a template for understanding the mechanisms mediating the secretion of CXCL10 and CXCL16. With Poly(I:C) treatment, Western-blot results manifested that the expression of phospho-NF-kB P65 and phospho-IRF3 apparently increased in a time-dependent manner (Fig. 5a), which 


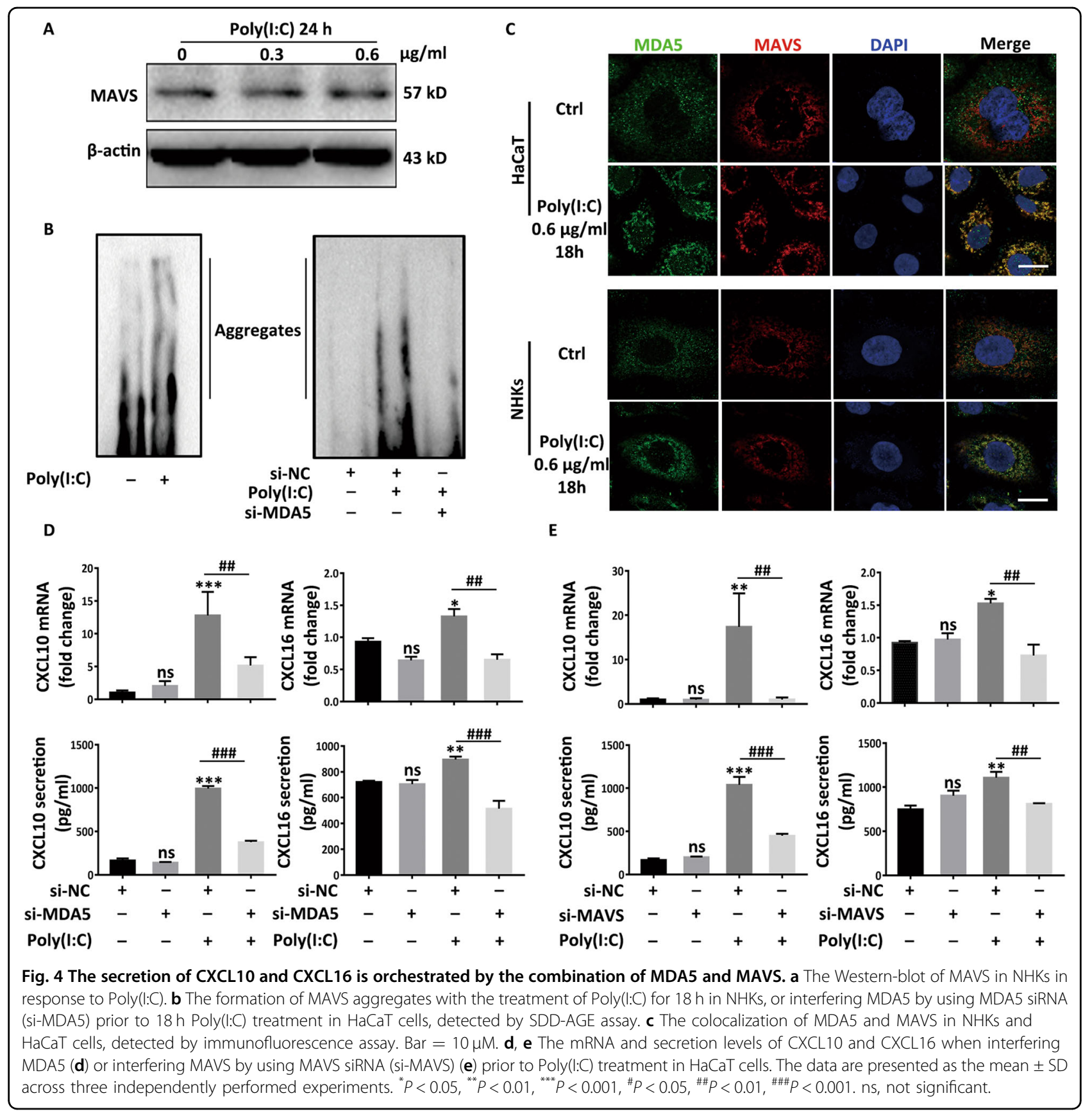

were diminished with the pretreatment of siRNA targeting MDA5 in $\mathrm{HaCaT}$ cells (Fig. 5b). Similarly, impeding MAVS also repressed the activation of NF-kB and IRF3 (Fig. 5c). Next, we silenced NF-kB and IRF3 by using corresponding siRNA (si-NF- $\mathrm{kB}$ and si-IRF3) to investigate the alterations of chemokines in mRNA and secretion levels in response to Poly(I:C) (Fig. S5C-5D). Results showed that interfering either NF- $\mathrm{kB}$ or IRF3 drastically dampened expression of CXCL10 and CXCL16 at both mRNA and secretion levels (Fig. 5d, e). These data underpinned critical roles of NF-kB and IRF3 in CXCL10 and CXCL16 secretion in the downstream of MAVS in keratinocytes.

MDA5-mediated IFN- $\beta$ is central to the secretion of CXCL10 while CXCL16 is directly regulated by IRF3

In $\mathrm{HaCaT}$ cells, we further determined whether the secretion of CXCL10 and CXCL16 is mediated by IFN- $\beta$ in response to Poly(I:C) stimulation. We proved Poly(I:C) potentiated the transcription of IFN- $\beta$ in a dose-dependent way as shown by qRT-PCR assay and promoted the secretion of IFN- $\beta$ in a time and concentration-dependent 


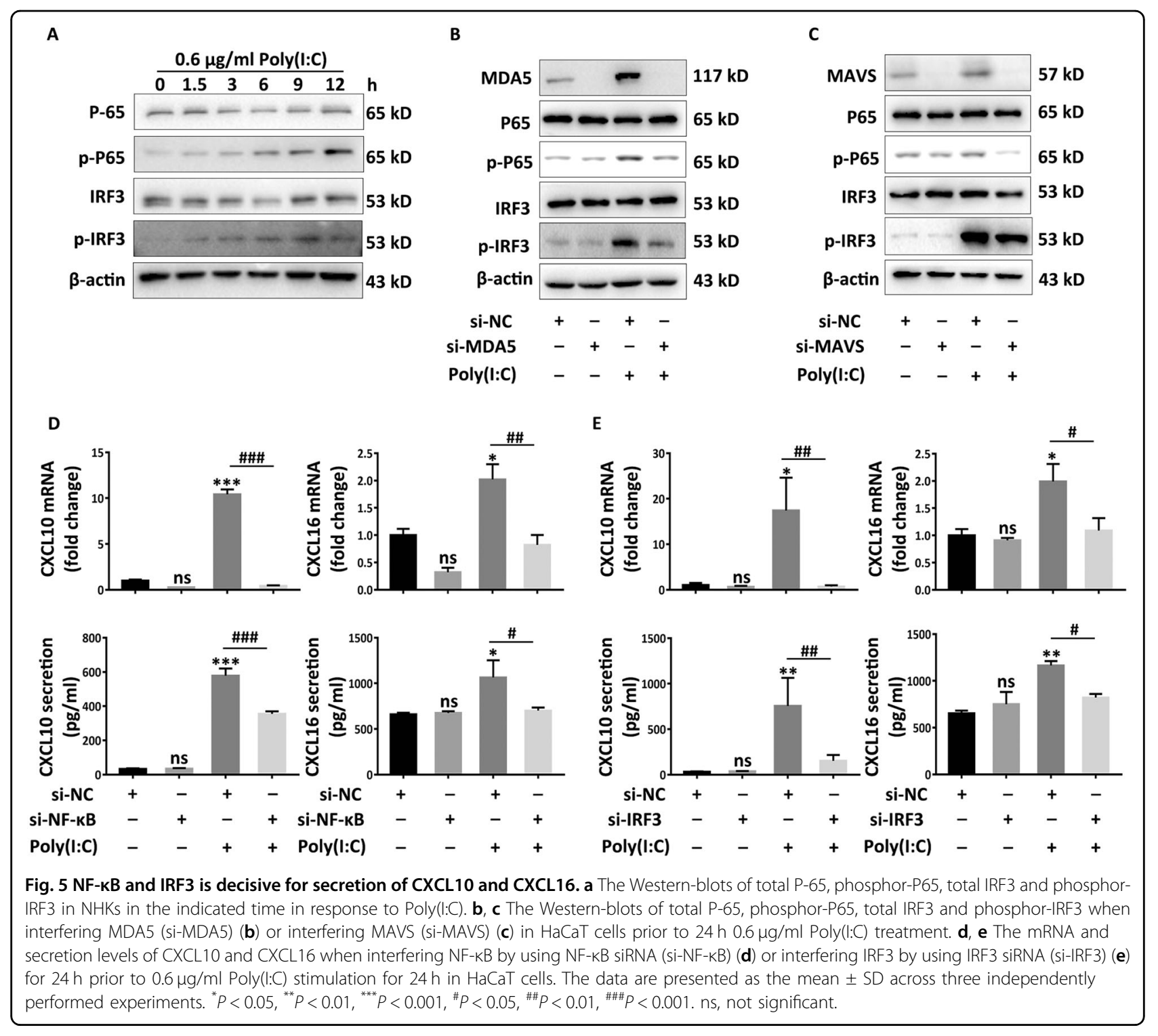

manner (Fig. 6a). Interfering any of MDA5, MAVS, NF$\kappa B$, and IRF3 could potently attenuate IFN- $\beta$ secretion (Fig. 6b). Then, after 24h Poly(I:C) treatment in $\mathrm{HaCaT}$ cells, we collected the supernatant (Sup) to treat $\mathrm{HaCaT}$ cells for $24 \mathrm{~h}$. After subtracting the basic CXCL10 and CXCL16, we found the supernatant could elevate the secretion of CXCL10 but not CXCL16. While neutralizing IFN- $\beta$ using IFN- $\beta$ neutralizing antibody (Neu) in supernatant disappeared the effect of promoting CXCL10 (Fig. 6c). Previous study verified that JAK1-STAT1 signaling functions in the downstream of IFN- $\beta^{55}$. Consistently, we found the Poly(I:C)-stimulated supernatant could apparently promote the expression of MDA5 and activate JAK1-STAT1 signaling pathway featuring the upregulation of phospho-JAK1 and phospho-STAT1 (Fig. $6 \mathrm{~d}$, e). Either using IFN- $\beta$ neutralizing antibody
(Fig. 6d) in the supernatant or pretreating $\mathrm{HaCaT}$ cells with the inhibitor of JAK1, Tofacitinib (Tofa) (Fig. 6e), impeded the activation of JAK1-STAT1 signaling pathway, which indicated JAK1-STAT1 signaling pathway functioned in the downstream of IFN- $\beta$ and this pathway could trigger the expression of MDA5 to form a positive feedback loop (Fig. 6e). What's more, we discovered that recombined human IFN- $\beta$ (rh IFN- $\beta$ ) indeed promoted the CXCL10 rather than CXCL16 at both mRNA and secretion levels (Fig. 6f). Consistently, pretreatment with Tofacitinib nullified the elevated secretion of CXCL10 elicited by rh IFN- $\beta$ (Fig. 6f). To explore the mechanism underlying the regulation of the CXCL16, we screened the JASPAR database (http://jaspar.genereg.net) and found the -2000 to +1 region of the CXCL16 promoter has 2 predicted binding sites for IRF3 (Fig. S6). The ChIP results 


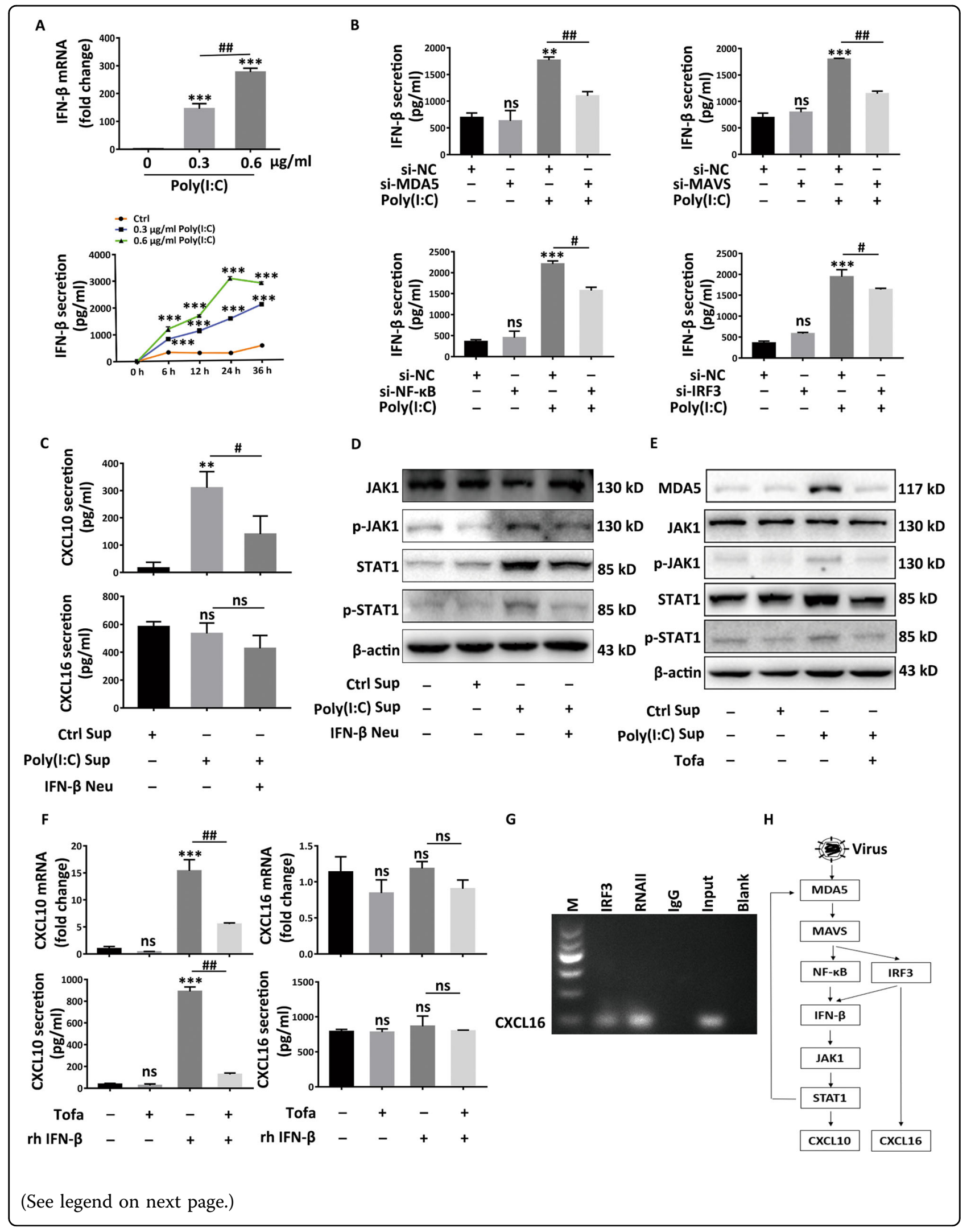


(see figure on previous page)

Fig. 6 MDA5-mediated IFN- $\beta$ is central to the secretion of CXCL10 while CXCL16 is directly regulated by IRF3. a The mRNA and secretion levels of IFN- $\beta$ in HaCaT cells in response to Poly(l:C). b The secretion levels of IFN- $\beta$ when interfering any of MDA5, MAVS, NF-kB, IRF3 prior to $24 \mathrm{~h}$ treatment of Poly(l:C) in HaCaT cells. c The secretion levels of CXCL10 and CXCL16 when treated with Poly(l:C)-pretreated supernatant (Sup) with or without neutralizing the IFN- $\beta$ by using the IFN- $\beta$ neutralizing antibody (Neu), detected by ELISA assay. $\mathbf{d}$, e The expression of total JAK, phosphorJAK, total STAT1, phosphor-STAT1 and MDA5 in HaCaT cells exposed to the Poly(l:C)-pretreated supernatant (Sup), and simultaneously with or without neutralizing IFN- $\beta$ (Neu) (d) or blocking JAK1-STAT1 pathway with the pretreatment of Tofacitinib (Tofa) (e). $\mathbf{f}$ The mRNA and secretion levels of CXCL10 and CXCL16 in HaCaT cells stimulated by recombined human IFN- $\beta$ (rh IFN- $\beta$ ) or pretreated with Tofacitinib (Tofa), detected by ELISA. g Chromatin immunoprecipitation assay (ChIP) of IRF3 to CXCL16 promoter after the HaCaT cells were treated by Poly(l:C) for $18 \mathrm{~h}$. $\mathbf{h}$ The diagram of the pathophysiological process in keratinocytes under the virus invasion. Data are presented as the mean \pm SD across three independently performed experiments. ${ }^{* *} P<0.01,{ }^{* * *} P<0.001,{ }^{\#} P<0.05,{ }^{\# \#} P<0.01$. ns, not significant.

also validated that IRF3 could bind directly to the promotor of CXCL16 and further promoted the transcription of CXCL16 under virus invasion (Fig. 6g). These data indicated MDA5-mediated IFN- $\beta$ is central to the secretion of CXCL10 in the JAK1-STAT1-dependent fashion, while CXCL16 is directly regulated by IRF3 via binding to the promotor of CXCL16 (Fig. 6h).

\section{Discussion}

In the present study, we initially manifested that the expression of MDA5 and anti-CMV IgM was upregulated in some progressive vitiligo. Then in vitro assays verified that virus infection, simulated by Poly(I:C), could promote the secretion of CXCL10 and CXCL16. Mechanistically, we demonstrated that IFN- $\beta$ mediated by the MDA5MAVS-NF- $K B / I R F 3$ signaling pathway orchestrated the secretion of CXCL10 via the JAK1-STAT1 pathway and MDA5-meidiated IRF3 transcriptionally induced the production of CXCL16 in keratinocytes under virus invasion. In short, we proved that targeting MDA5 signaling pathway has the potent therapeutic potential in retarding the aggravation of vitiligo under virus invasion.

Increasing evidences have indicated the correlation between virus infection and autoimmune diseases such as Coxsackie virus and type 1 diabetes $^{6}$, and the potential mechanisms embody adjuvant initiative effect of autoimmune reaction, molecule mimicry, bystander activation and epitope spreading ${ }^{56}$. Vitiligo, as an autoimmunityinvolved disease, is thought to correlate with virus infection $^{14-16,19-22}$. Recently, IFIH1, encoding MDA5 which is a virus-associated pattern recognition receptor (PRR), has been identified as a susceptible gene for vitiligo ${ }^{3}$. Herein, we found the positive rate and levels of anti-CMV IgM in the serum of progressive vitiligo patients were significantly higher than that in healthy controls. In addition, our data showed the expression of MDA5 and IFN- $\beta$ was enhanced in keratinocytes of vitiligo patients with positive anti-CMV IgM. As the sporadic evidences indicated, although MDA5 could also be activated by some selfderived dsRNA ${ }^{57,58}$ in vivo, the human evolves many crucial mechanisms to prevent the activation of IFIH1 via inhibiting the production of endogenous recognizable RNA $^{57-60}$. More importantly, given that the higher expression of MDA5 and IFN- $\beta$ is along with the positive anti-CMV IgM in some progressive vitiligo patients, we attribute the higher expression of MDA5 in the vitiligo patients with positive anti-CMV IgM to virus infection. All these above strengthen that virus infection is involved in some progressive vitiligo pathogenesis and indicate that MDA5 plays a key role in vitiligo under virus invasion.

MDA5, as a cytosolic virus sensor, contributes significantly to the elimination of virus in end-organs not only through the induction of antiviral interferons and other pro-inflammatory cytokines, but also by facilitating cell death ${ }^{5}$. Meanwhile, the inappropriate gain-offunction of MDA5 could efficiently eradicate virus, but at the cost of increasing incidence to suffer autoimmune disease such as type-1 diabetes (T1D) ${ }^{61}$ and systemic lupus erythematosus (SLE) ${ }^{62}$. This argues against that the persistent viral infection per se induces autoimmunity and indicates that MDA5-mediated aberrant skin immunity functions prominently,63. Herein, we highlighted the impacts of MDA5 on the dysfunctional local immune microenvironment, particularly on the secretion promotion of CXCL10 and CXCL16 under virus invasion in keratinocytes. Previous studies have in succession clarified that CXCL10 ${ }^{43}$ and CXCL16 $6^{45}$ play key roles in the trafficking of melanocyte-specific autoreactive $\mathrm{CD}^{+} \mathrm{T}$ cells from periphery blood to skin, which contributed significantly to melanocytes death in vitiligo. Specifically, the in vivo assay conducted in a mouse vitiligo model verified that, mice received $\mathrm{Cxcr3}^{-1-} \mathrm{T}$ cells develop minimal depigmentation, as do mice lacking $\mathrm{Cxcl10}$ or treated with CXCL10-neutralizing antibody. In addition, CXCL10 neutralization treatment in vitiligo mice with the established and widespread depigmentation induces repigmentation $^{43}$. Besides, functional study unveiled that the IFN- $\gamma$-JAK-STAT signaling axis is required for CXCL10 secretion in epidermis of vitiligo ${ }^{44}$ and blockade of this pathway using tofacitinib or ruxolitinib which are all JAK inhibitor rapidly induces the repigmentation of 
vitiligo $^{64,65}$. With respect to the important roles of CXCL16 in vitiligo, we previously demonstrated that compared with nonlesional and healthy control skin, more $\mathrm{CXCR}^{+} \mathrm{CD}^{+} \mathrm{T}$ cells are located at the basal epidermis and dermis of perilesions from vitiligo patients, which is consistent with the higher content of CXCL16 in epidermis. Besides, in vitro assay verified that neutralizing CXCL16 in the supernatant of $\mathrm{H}_{2} \mathrm{O}_{2}$-treated primary keratinocytes significantly decreases the migration of $\mathrm{CD}^{+} \mathrm{T}$ cells sorted from vitiligo patients ${ }^{45}$. All these above further underscore the significance of MDA5mediated chemokines in the pathogenesis of vitiligo. In addition, Previous studies have proved that IFN- $\gamma$ signature $^{44,49}$ and oxidative stress ${ }^{45}$ play key parts in the secretion of chemokines in keratinocytes. Our data further complement the specific mechanisms of chemokines secretion from keratinocytes during the process of melanocytes death and extend the underlying mechanism by which virus infection engages in autoimmune disease.

Notably, previous studies also emphasized the roles of IFN- $\beta$ in reinforcing and bridging the innate and adaptive immunology such as promoting dendritic cells (DCs) maturation, skewing of $\mathrm{TH} 1$ polarization and reactivation of CTLs (cytotoxic T lymphocyte) ${ }^{41,66}$. Besides, MDA5 belongs to the RIG-I-like receptor family (RLR family) which could initiate the production of pro-inflammatory factors including IL-6, IL-8, IL-23, and TNF- $\alpha^{31,67,68}$. Hence, whether other functions of MDA5 participate in the pathogenesis of vitiligo in response to virus invasion is worthy to be explored.

Mechanistically, we demonstrated that MDA5 exerted its function by inducing the formation of MAVS aggregates after the colocalization of MDA5 and MAVS in keratinocytes. This result parallels the canonic MDA5 signaling pathway that, MDA5 binds to viral dsRNA through the C-terminal RD domain and sequentially the exposed $\mathrm{N}$-terminal CARD domains interact with MAVS which rapidly forms the prion-like aggregates $^{36}$. What's more, our data validated that the Poly(I:C)-stimulated keratinocytes supernatant had no impacts on the CXCL16 secretion and IRF3 could directly bind to the promotor of CXCL16 in response to virus. Combined with our previous finding that NF- $k B$ P65 could directly mediate the transcription of CXCL16 in keratinocytes through directly binding to the promotor of CXCL16 ${ }^{45}$, we draw a conclusion that CXCL16 is mainly elaborated by IRF3 and NF-kB in the downstream of MAVS under virus infection. As for CXCL10, we exhibited that it was orchestrated to a large extent by IFN- $\beta$ which was induced by the MDA5 canonic pathway. Notably, another study underscored that Hantaan virus, the pathogen of hemorrhagic fever with renal syndrome (HFRS), promotes the secretion of CXCL10 by facilitating $\mathrm{NF}-\mathrm{kB}$ and IRF7 to directly bind to the promotor of
CXCL10 in the downstream of MDA $5^{69}$. This supports that NF- $\mathrm{kB}$ might mediate the secretion of CXCL10 not merely through IFN- $\beta$ but also via prompting the transcription of CXCL10 directly. Moreover, our data exhibited that IFN- $\beta$ could significantly prompt the expression of MDA5 via the JAK1-STAT1 signaling pathway, thus forming a positive feedback loop to aggravate this pathogenesis.

The present study deciphers that MDA5 could exacerbate vitiligo by inducing the secretion of chemokines in keratinocytes under virus invasion. Even so, the specific causal relationship are still difficult to solidly identify due to the complex interaction between virus infection and vitiligo such as the "hit and run" hypothesis of virus ${ }^{6,56}$. Hence, identifying the pathological evidence of specific virus or the solid causative link with virus infection in vitiligo warrants further investigation. In addition, based on our present data, in vivo assay should be further performed to explore the remarkable contribution of virus invasion in the exacerbation of vitiligo via the MDA5-chemokines pathway. Aiming at individualized treatment, we think our research could provide the evidence to the MDA5-targeting therapy in vitiligo under virus invasion.

\begin{abstract}
Acknowledgements
This work was supported by the National Natural Science Foundation of China (No. 81930087, No. 91742201, No. 81625020 and No. 81773315). We thank all the patients and the healthy volunteers who consented to provide their skin and blood specimens.
\end{abstract}

Conflict of interest

The authors declare that they have no conflict of interest.

Publisher's note

Springer Nature remains neutral with regard to jurisdictional claims in published maps and institutional affiliations.

Supplementary Information accompanies this paper at (https://doi.org/ 10.1038/s41419-020-2665-z).

Received: 22 December 2019 Revised: 8 April 2020 Accepted: 8 April 2020 Published online: 12 June 2020

\footnotetext{
References

1. van den Boorn, J. G. et al. Autoimmune destruction of skin melanocytes by perilesional t cells from vitiligo patients. J. Investigative Dermatol. 129, 2220-2232 (2009).

2. Frisoli, M. L. \& Harris, J. E. Vitiligo: mechanistic insights lead to novel treatments. J. Allergy Clin. Immunol. 140, 654-662 (2017).

3. Spritz, R. A. \& Andersen, G. H. L. Genetics of Vitiligo. Dermatol. Clin. 35, 245-255 (2017).

4. Richardson, S. J., Willcox, A., Bone, A. J., Foulis, A. K. \& Morgan, N. G. The prevalence of enteroviral capsid protein vp1 immunostaining in pancreatic islets in human type 1 diabetes. Diabetologia 52, 1143-1151 (2009).

5. Dou, Y., Yim, H. C., Kirkwood, C. D., Williams, B. R. \& Sadler, A. J. The innate immune receptor MDA5 limits rotavirus infection but promotes cell death and pancreatic inflammation. EMBO J. 36, 2742-2757 (2017).

6. Op, D. B. A. \& Eizirik, D. L. Viral infections in type 1 diabetes mellitus-why the beta cells? Nat. Rev. Endocrinol. 12, 263-273 (2016).
} 
7. Balandraud, N. et al. Epstein-Barr virus load in the peripheral blood of patients with rheumatoid arthritis: accurate quantification using real-time polymerase chain reaction. Arthritis Rheumatism 48, 1223-1228 (2003).

8. Lunemann, J. D. et al. Increased frequency of EBV-specific effector memory CD8 $+T$ cells correlates with higher viral load in rheumatoid arthritis. J. Immunol. 181, 991-1000 (2008).

9. Scotet, E. et al. T cell response to Epstein-Barr virus transactivators in chronic rheumatoid arthritis. J. Exp. Med. 184, 1791-1800 (1996).

10. Sultanova, A. et al. Association of active human herpesvirus-6 (HHV-6) infection with autoimmune thyroid gland diseases. Clin. Microbiol. Infect. 23, 50-51 (2017).

11. Ferrari, S. M., Fallahi, P., Antonelli, A.. \& Benvenga, S. Environmental issues in thyroid diseases. Front Endocrinol. 8, https://doi.org/10.3389/fendo.2017.00050 (2017).

12. Laberge, G. et al. Early disease onset and increased risk of other autoimmune diseases in familial generalized vitiligo. Pigment Cell Res. 18, 300-305 (2005).

13. Alkhateeb, A., Fain, P. R., Thody, A., Bennett, D. C. \& Spritz, R. A. Epidemiology of vitiligo and associated autoimmune diseases in Caucasian probands and their families. Pigment Cell Res. 16, 208-214 (2003).

14. Grimes, P. E., Sevall, J. S. \& Vojdani, A. Cytomegalovirus DNA identified in skin biopsy specimens of patients with vitiligo. J. Am. Acad. Dermatol. 35, 21-26 (1996).

15. El-Serag, H. Extrahepatic manifestations of hepatitis $C$ among United States male veterans. Hepatology 36, 1439-1445 (2002).

16. Erf, G. F., Bersi, T. K., Wang, X., Sreekumar, G. P. \& Smyth, J. J. Herpesvirus connection in the expression of autoimmune vitiligo in Smyth line chickens. Pigment Cell Res. 14, 40-46 (2001).

17. Yamamoto, T. \& Nishioka, K. Vitiligo vulgaris associated with hepatitis C virus. J. Dermatol. 27, 416-417 (2000)

18. Kumar, A., Dogra, S., Gupta, A. \& Suri, D. Treatment of vitiligo with antiretroviral therapy in a child with HIV infection. AIDS 30, 1309-1310 (2016).

19. Tsuboi, H., Yonemoto, K. \& Katsuoka, K. Vitiligo with inflammatory raised borders with hepatitis C virus infection. J. Dermatol. 33, 577-578 (2006).

20. Jung, Y. S., Lee, J. H., Kim, G. M. \& Bae, J. M. Reactivation of herpes simplex virus secondary to excimer laser treatment in a patient with vitiligo. ANN Dermatol. 29, 493 (2017).

21. Morita, C., Yanase, T., Shiohara, T. \& Aoyama, Y. Aggressive treatment in paediatric or young patients with drug-induced hypersensitivity syndrome (DiHS)/ drug reaction with eosinophilia and systemic symptoms (DRESS) is associated with future development of type III polyglandular autoimmune syndrome. BMJ Case Rep. 2018-225528 https://casereports.bmj.com/content/2018/bcr2018-225528 (2018)

22. Lonowski, S., Hau, J. \& Worswick, S. Vitiligo: a potential autoimmune sequela of DRESS syndrome. Brit. J. Dermatol. 175, 642-644 (2016).

23. Jin, Y., Andersen, G., Santorico, S. A. \& Spritz, R. A. Multiple functional variants of $|F| H 1$, a gene involved in triggering innate immune responses, protect against vitiligo. J. Invest Dermatol 137, 522-524 (2017).

24. Jin, $Y$. et al. Genome-wide association analyses identify 13 new susceptibility loci for generalized vitiligo. NAT Genet. 44, 676-680 (2012).

25. Shen, C. et al. Genetic susceptibility to vitiligo: GWAS approaches for identifying vitiligo susceptibility genes and loci. Front. Genet. 7, 3 (2016).

26. Majde, J. A. Viral double-stranded RNA, cytokines, and the flu. J. Interferon Cytokine Res. 20, 259-272 (2000).

27. Dias Junior, A. G., Sampaio, N. G. \& Rehwinkel, J. A Balancing Act: MDA5 in Antiviral Immunity and Autoinflammation. Trends Microbiol. 27, 75-85 (2019).

28. Kato, $\mathrm{H}$. et al. Length-dependent recognition of double-stranded ribonucleic acids by retinoic acid-inducible gene-I and melanoma differentiationassociated gene 5. J. Exp. Med. 205, 1601-1610 (2008).

29. Kasumba, D. M. \& Grandvaux, N. Therapeutic targeting of RIG-I and MDA5 might not lead to the same rome. Trends Pharmocol. Sci. 40, 116-127 (2019).

30. Gorman, J. A. et al. The A946T variant of the RNA sensor IFIH1 mediates an interferon program that limits viral infection but increases the risk for autoimmunity. NAT Immunol. 18, 744-752 (2017).

31. Zhu, $\mathrm{H}$. et al. RIG-I antiviral signaling drives interleukin-23 production and psoriasis-like skin disease. EMBO Mol. Med. 9, 589-604 (2017).

32. Lincez, P. J., Shanina, I. \& Horwitz, M. S. Reduced expression of the MDA5 Gene IFIH1 prevents autoimmune diabetes. DIABETES 64, 2184-2193 (2015).

33. Funabiki, M. et al. Autoimmune disorders associated with gain of function of the intracellular sensor MDA5. Immunity 40, 199-212 (2014).

34. Wu, B. et al. Structural basis for dsRNA recognition, filament formation, and antiviral signal activation by MDA5. Cell 152, 276-289 (2013).
35. Chow, K. T., Gale, M. J. \& Loo, Y. M. RIG-I and other RNA sensors in antiviral immunity. Annu. Rev. Immunol. 36, 667-694 (2018).

36. Hou, F. et al. MAVS forms functional prion-like aggregates to activate and propagate antiviral innate immune response. Cell 146, 448-461 (2011).

37. Liu, S. et al. Phosphorylation of innate immune adaptor proteins MAVS, STING, and TRIF induces IRF3 activation. Science 347, a2630 (2015).

38. Dai, T. et al. FAF1 Regulates antiviral immunity by inhibiting MAVS but is antagonized by phosphorylation upon viral infection. Cell Host Microbe 24, 776-790 (2018)

39. Stone, A., Green, R., Wilkins, C., Hemann, E. A. \& Gale, M. J. RIG-H-like receptors direct inflammatory macrophage polarization against West Nile virus infection. Nat. Commun. 10, 3649 (2019).

40. Yamada, S. et al. RIG-I-Like receptor and toll-like receptor signaling pathways cause aberrant production of inflammatory cytokines/chemokines in a severe fever with thrombocytopenia syndrome virus infection mouse model. J. Virol. 92, e2217-e2246 (2018).

41. Sprokholt, J. K. et al. RIG--like receptor triggering by dengue virus drives dendritic cell immune activation and TH1 differentiation. J. Immunol. 198, 4764-4771 (2017).

42. Blank, T. et al. Brain endothelial- and epithelial-specific interferon receptor chain 1 drives virus-induced sickness behavior and cognitive impairment. Immunity 44, 901-912 (2016).

43. Rashighi, M. et al. CXCL10 is critical for the progression and maintenance of depigmentation in a mouse model of vitiligo. Sci. Transl. Med. 6, 223r (2014).

44. Rashighi, M. \& Harris, J. E. Interfering with the IFN-gamma/CXCL10 pathway to develop new targeted treatments for vitiligo. Ann. Trans/. Med. 3, 343 (2015).

45. Li, S. et al. Oxidative stress drives CD8 + T-cell skin trafficking in patients with vitiligo through CXCL16 upregulation by activating the unfolded protein response in keratinocytes. J. Allergy Clin. Immunol. 140, 177-189 (2017).

46. Jia, J. et al. Cytomegalovirus infection may trigger adult-onset still's disease onset or relapses. Front. Immunol. 10, 898 (2019).

47. Feily, A. Vitiligo extent tensity index (VETI) score: a new definition, assessment and treatment evaluation criteria in vitiligo. Dermatol. Pr. Concept 4, 81-84 (2014).

48. Del Toro Duany, Y., Wu, B. \& Hur, S. MDA5-filament, dynamics and disease Curr. Opin. Virol. 12, 20-25 (2015).

49. Harris, J. E. et al. A mouse model of vitiligo with focused epidermal depigmentation requires IFN-gamma for autoreactive CD8(+) T-cell accumulation in the skin. J. Invest. Dermatol. 132, 1869-1876 (2012).

50. Richmond, J. M. et al. Keratinocyte-derived chemokines orchestrate T-cell positioning in the epidermis during vitiligo and may serve as biomarkers of disease. J. Invest. Dermatol. 137, 350-358 (2017).

51. Zhang, L. et al. Circulating CCL20: a potential biomarker for active vitiligo together with the number of Th1/17 cells. J. Dermatol. Sci. 93 92-100 (2019).

52. Chen, $X$. et al. Oxidative stress-induced IL-15 trans-presentation in keratinocytes contributes to CD8+ T cells activation via JAK-STAT pathway in vitiligo. Free Radical Bio Med. 139, 80-91 (2019).

53. Rezk, A. F. et al. Misbalanced CXCL12 and CCL5 chemotactic signals in vitiligo onset and progression. J. Invest. Dermatol. 137, 1126-1134 (2017).

54. Seth, R. B., Sun, L., Ea, C. \& Chen, Z. J. Identification and characterization of MAVS, a mitochondrial antiviral signaling protein that activates NF-KB and IRF3. Cell 122, 669-682 (2005).

55. Eizirik, D. L. \& Op, D. B. A. Coxsackievirus and type 1 diabetes mellitus: the Wolf's footprints. Trends Endocrinol. Metab. 29, 137-139 (2018).

56. Munz, C., Lunemann, J. D., Getts, M. T. \& Miller, S. D. Antiviral immune responses: triggers of or triggered by autoimmunity? Nat. Rev. Immunol. 9, 246-258 (2009)

57. Dhir, A. et al. Mitochondrial double-stranded RNA triggers antiviral signalling in humans. Nature 560, 238-242 (2018).

58. Wang, Y. \& Colonna, M. RNA exosomes keep endogenous RNA under the radar. Nat. Immunol. 15, 830-831 (2014).

59. Nakahama, T. et al. ADAR1-mediated RNA editing is required for thymic selftolerance and inhibition of autoimmunity. EMBO Rep. 19, e46303 (2018).

60. Chung, $H$. et al. Human ADAR1 prevents endogenous RNA from triggering translational shutdown. Cell 172, 811-824 (2018).

61. Shigemoto, T. et al. Identification of loss of function mutations in human genes encoding RIG-I and MDA5: implications for resistance to type I diabetes. J. Biol. Chem. 284, 13348-13354 (2009). 
62. Liu, Z. \& Davidson, A. Taming lupus-a new understanding of pathogenesis is leading to clinical advances. Nat. Med. 18, 871-882 (2012).

63. Dotta, F. et al. Coxsackie B4 virus infection of beta cells and natural killer cell insulitis in recent-onset type 1 diabetic patients. Proc. Natl Acad. Sci. USA 104 5115-5120 (2007).

64. Craiglow, B. G. \& King, B. A. Tofacitinib citrate for the treatment of vitiligo: a pathogenesis-directed therapy. JAMA Dermatol. 151, 1110-1112 (2015).

65. Harris, J. E. et al. Rapid skin repigmentation on oral ruxolitinib in a patient with coexistent vitiligo and alopecia areata (AA). J. Am. Acad. Dermatol. 74, 370-371 (2016).
66. Yang, $X$. et al. Targeting the tumor microenvironment with interferon- $\beta$ bridges innate and adaptive immune responses. Cancer Cell. 25, 37-48 (2014).

67. Wang, S., Liu, D., Jin, R., Zhu, Y. \& Xu, A. Differential responses of normal human melanocytes to intra- and extracellular dsRNA. DNA Cell Biol. 34, 391-399 (2015).

68. Molony, R. D. et al. Aging impairs both primary and secondary RIG-I signaling for interferon induction in human monocytes. Sci. Signal 10, eaan2392 (2017).

69. Zhang, Y. et al. Hantaan virus infection induces CXCL10 expression through TLR3, RIG-I, and MDA-5 pathways correlated with the disease severity. Mediat. Inflamm. 2014, 1-11 (2014). 